\title{
Molecular, cytogenetic, and morphological features of primary octoploid triticale
}

\author{
Evtushenko E.V. ${ }^{1 *}$, Lipikhina Yu.A ${ }^{1}$., Stepochkin P.I. ${ }^{2}$, Vershinin A.V. ${ }^{1}$ \\ ${ }^{1}$ Institute of Molecular and Cellular Biology, SB RAS, Novosibirsk, Russia \\ ${ }^{2}$ Institute of Cytology and Genetics, SB RAS, Novosibirsk, Russia \\ *e-mail:evt@mcb.nsc.ru
}

Triticale ( $\times$ Triticosecale Wittmack) is artificial intergeneric hybrid that combine genomes of wheat (Triticum spp.) and rye (Secale spp.) Triticales are a convenient biologic model for study of genetic and epigenetic changes associated with allopolyploidization in Triticeae. Remote hybridization of plants induces genomic rearrangements, which may cause the elimination of chromosomes of one of the parents, chromosome number reduction owing to fusions and rearrangements, and gene expression modification. Faithful chromosome segregation in mitosis and meiosis depends on the absence of aberrations in centromere function. The centromere structure is determined by the centromeric histone $\mathrm{H} 3$ variant, $\mathrm{CENH} 3$. We studied the CENH3 structure and expression in wheat-rye allopolyploids with various ploidies and their parental forms. The cytogenetic analysis included the comparison of in situ hybridization patterns with the highly repetitive pSc200 DNA probe and centromere measurement in the allopolyploids and parental forms. Allopolyploids (genome AABBDDRR) were synthesized by crossing isogenic line Triple Dirk D (Triticum aestivum L.) (AABBDD) with rye (Secale cereale L.) cv. Korotkostebelnaya 69 (RR). Hybrids of generations $\mathrm{S}_{1}$ through $\mathrm{S}_{5}$ were analyzed. The coding sequences of $\alpha C E N H 3 \mathrm{~N}$-terminal tail from hybrid $\mathrm{S}_{2}-\mathrm{S}_{5}$ plants with different chromosome numbers $(2 n=42-56)$ and their parental forms were amplified with specific primers, and their analysis revealed nonsynonymous nucleotide substitutions characteristic of wheat and rye. The expression level of CENH3 copies with SNPs characteristic of rye CENH3 increased from generation $\mathrm{S}_{2}(11 \%)$ to generation $\mathrm{S}_{5}(57 \%)$. Fluorescent in situ hybridization analysis (FISH) with the highly repetitive DNA probe pSc200 showed identical hybridization patterns in hybrids having different numbers of chromosomes $(2 n=42-56)$, which were also the same in the parental rye variety Korotkostebelnaya 69 . Stable octoploid triticales $(2 n=56)$ preserved the rye chromosome set throughout generations $\mathrm{S}_{2}-\mathrm{S}_{5}$, but the plants showed phenotypic differences.

Acknowledgements: This study was supported by the Russian Fundamental Research Program, projects 0310-2019-0003, 0324-2019-0039, and the Russian Foundation for Basic Research, project 18-54-00013a. 